\title{
Análise da estrutura vertical do Balanço de Energia Cinética Turbulenta durante a ocorrência de eventos de intermitência global
}

\author{
Analysis of the vertical structure of Turbulent Kinetic Energy Balance for the \\ occurrence of intermittent turbulent events
}

\author{
Thaís Freitas Dill $^{1}$, Adriano Battisti ${ }^{1}$, Viviane da Silva Guerra ${ }^{1}$, Felipe Denardin Costa ${ }^{2}$, Luiz \\ Eduardo Medeiros ${ }^{2}$ \\ ${ }^{1}$ Universidade Federal de Santa Maria, Santa Maria, Brasil \\ ${ }^{2}$ Universidade Federal do Pampa, Alegrete, Brasil
}

\begin{abstract}
Resumo
Este trabalho tem como objetivo a analise da estrutura vertical dos termos da equação de balanço de ECT durante a ocorrência de eventos intermitentes gerados em superfície e que se propagam para cima. Afim de verificar quais os termos dominantes e qual o papel de cada termo durante a ocorrência desses eventos. Utilizou-se dados do experimento FLOSSII, coletados em sete níveis verticais $(1 \mathrm{~m}, 2 \mathrm{~m}, 5 \mathrm{~m}, 10 \mathrm{~m}, 15 \mathrm{~m}, 20 \mathrm{~m}$ e $30 \mathrm{~m}$ ) e possuem uma frequência de amostragem de $60 \mathrm{~Hz}$. O período de estudo compreende entre 20 de Novembro de 2002 a 02 de Abril de 2003 e desse total, neste trabalho, foram analisados 108 noites. A maior parte dos eventos gerados em superfície (EB) são muito fracos em comparação com os eventos gerados no topo da camada da torre e se propagam para baixo. As análises feitas através da estrutura vertical da turbulência e do balanço de ECT para os EB's mostraram que o termo dominante próximo a superfície é o de produção mecânica. Dessa forma, com o aumento do cisalhamento em superfície ocorre a geração de turbulência e o termo de transporte atua "carregando" a turbulência para os níveis mais altos da atmosfera.
\end{abstract}

Palavras-chave: Intermitência, camada limite estável, cisalhamento, turbulência, propagação vertical.

\begin{abstract}
This study aims to analyze the vertical structure of the terms of the ECT balance equation for the occurrence of intermittent events generated in surface and propagate upward. In order to verify that the dominant terms and the role of each term during such events. It was used FLOSSII experimental data collected in seven vertical levels $(1 \mathrm{~m}, 2 \mathrm{~m}, 5 \mathrm{~m}, 10 \mathrm{~m}, 15 \mathrm{~m}, 20 \mathrm{~m}$ and 30 m) and have a sampling frequency of $60 \mathrm{~Hz}$. The study period comprises between 20 November 2002 to 02 April 2003 and of that total, this study, we analyzed 108 nights. The majority of events generated surface (EB) are very weak compared to the events generated at the top of the tower layer and propagate down. The analyzes made by vertical turbulence structure and ECT balance for the EB's showed that the dominant term near surface is the mechanical production. Thus, with increasing shear surface, producing turbulence and consequently, the transport of works term "loading" turbulence to higher levels of the atmosphere.
\end{abstract}

Keywords: Intermittency, stable boundary layer, shear, turbulence, vertical propagation. 


\section{Introdução}

A Camada Limite Estável (CLE) se forma durante o período noturno em decorrência do resfriamento radiativo a partir da superfície. Nestas condições o escoamento se torna menos turbulento e o cisalhamento do vento é o único agente responsável pela geração de turbulência. Em noites de vento fraco e com grande perda radiativa é geralmente observado a supressão da turbulência em quase todas as suas escalas. Entretanto, de forma abrupta e imprevisível é comum observar a ocorrência de períodos de intensa atividade turbulenta e comumente logo após a sua ocorrência a turbulência é suprimida novamente. A alternância entre períodos no qual a turbulência é quase que totalmente suprimida e períodos de intensa mistura é conhecida como intermitência global (Mahrt, 1999).

Por não existe um padrão claro para a ocorrência do fenômeno da intermitência, identificar as causas que geram estes eventos é um dos principais desafios no entendimento do comportamento do escoamento na CLE, seja no âmbito experimental ou teórico. Alguns estudam indicam que a natureza física desses eventos estão associados aos níveis mais elevados da CLE e se propagam posteriormente para baixo chegando a serem observados próximo a superfície. Como por exemplo, jatos de baixos níveis, ondas de gravidade e instabilidades de Kelvin-Helmhontz, ou devido à características do sítio experimental (Mahrt, 1999; Cuxart et al., 2000; Banta et al., 2002; Sun et al., 2004; Banta et al., 2006, entre outros).

Dessa forma, este trabalho tem como objetivo a analise da estrutura vertical dos termos da equação de balanço de ECT durante a ocorrência de eventos intermitentes, com foco nos eventos gerados em superfície e que se propagam para cima. Afim de verificar quais os termos dominantes e qual o papel de cada termo durante a ocorrência desses eventos.

\section{Metodologia}

A CLE possui forte complexidade no que diz respeito à turbulência e uma forma de descrever o comportamento da turbulência na CLE é através da equação de balanço da ECT. Dessa forma, assumindo homogeneidade horizontal e desprezando os efeitos de advecção, a equação de balanço de ECT pode ser escrita como (Stull, 1988):

$$
\begin{array}{r}
\frac{\partial \bar{e}}{\partial t}=-\overline{u^{\prime} w^{\prime}} \frac{\partial u}{\partial z}-\overline{v^{\prime} w^{\prime}} \frac{\partial v}{\partial z}+\frac{g}{\Theta} \overline{w^{\prime} \theta^{\prime}}- \\
\frac{\partial}{\partial z}\left[\left(\overline{w^{\prime} e^{\prime}}\right)-\frac{\overline{p^{\prime} w^{\prime}}}{\rho_{0}}\right]-\epsilon .
\end{array}
$$

Os termos da equação (1) descrevem processos físicos que geram turbulência e indicam produção, destruição, ou transporte de turbulência. O termo I representa a tendência ou armazenamento da ECT com tempo. O segundo termo representa a produção ou destruição da turbulência devido ao fluxo na forma de calor. $\mathrm{O}$ termo III é a produção mecânica de ECT, e o quarto termo é de transporte turbulento de ECT. O transporte devido as flutuações de pressão é representado pelo termo V. O termo VI é a dissipação viscosa da energia cinética turbulenta que é convertida em calor.

Análise do comportamento dos termos da ECT durante a ocorrência de eventos intermitentes foi feita utilizando dados do experimento FLOSSII (Fluxos Sobre Superfícies com Neve, do inglês Fluxes Over Snow Surfaces), realizado em North Park ao sul de Walden, no estado do Colorado, nos Estados Unidos (40,8 ${ }^{\circ} \mathrm{N}, 106,3$ $\left.{ }^{\circ} \mathrm{W}\right)$. A torre micrometeorológica possui $34 \mathrm{~m}$, localizada em uma área de pastagem entre duas cadeias de montanhas, com dados coletados em sete níveis verticais (1 m, $2 \mathrm{~m}, 5 \mathrm{~m}, 10 \mathrm{~m}, 15 \mathrm{~m}, 20 \mathrm{~m}$ e $30 \mathrm{~m}$ ) e possuem uma frequência de amostragem de $60 \mathrm{~Hz}$ (Mahrt e Vickers, 2006; Mahrt, 2010, entre outros). O experimento foi realizado no período compreendido entre 20 de Novembro de 2002 a 02 de Abril de 2003 (inverno na região) e desse total, neste trabalho, foram analisados 108 noites. Esses dados noturnos possuem $10 \mathrm{~h}$ de duração, com início as 20 h e término às $6 \mathrm{~h}$, horário local. Durante a realização do projeto, geralmente a superfície, e os instrumentos da torre, eram cobertos por uma fina camada de gelo (Mahrt, 2010).

A metodologia utilizada para a seleção dos eventos intermitentes se baseou primeiramente levando em consideração o desvio padrão da velocidade vertical do vento $\left(\sigma_{w}\right)$. Cada noite foi analisada separadamente, e os eventos foram caracterizados levando em consideração a definição de intermitência global de Mahrt (1999) e Coulter e Doran (2002), onde ocorrem grandes picos de turbulência seguidos de períodos calmos. Foram considerados eventos intermitentes, toda a atividade turbulenta isolada, temporalmente, $\operatorname{com} \sigma_{w} \geq 0,25$. A partir deste critério duas classes para caracterizar os eventos foram estabelecidas: Aqueles que se originaram em superfície (níveis de 1 e $2 \mathrm{~m}$ da torre) e se propagaram para os níveis mais altos, denominados a partir daqui como Eventos de Baixo (EB); Os originados nos níveis mais altos e se propagaram para a superfície, denominados Eventos de Cima (EC).

A distinção entre os dois tipos de eventos é feita apenas considerando o sentido de propagação do $\sigma_{w}$ (Figura 1). A partir da análise da série temporal se observa o momento do início do evento em um determinado nível e qual o sentido, para cima ou para baixo, que irá ocorrer a propagação, conforme mostrado na Figura 1. Cabe ressaltar, que esta análise é qualitativa, sendo que não foi utilizado nenhum método objetivo, como foi utili- 


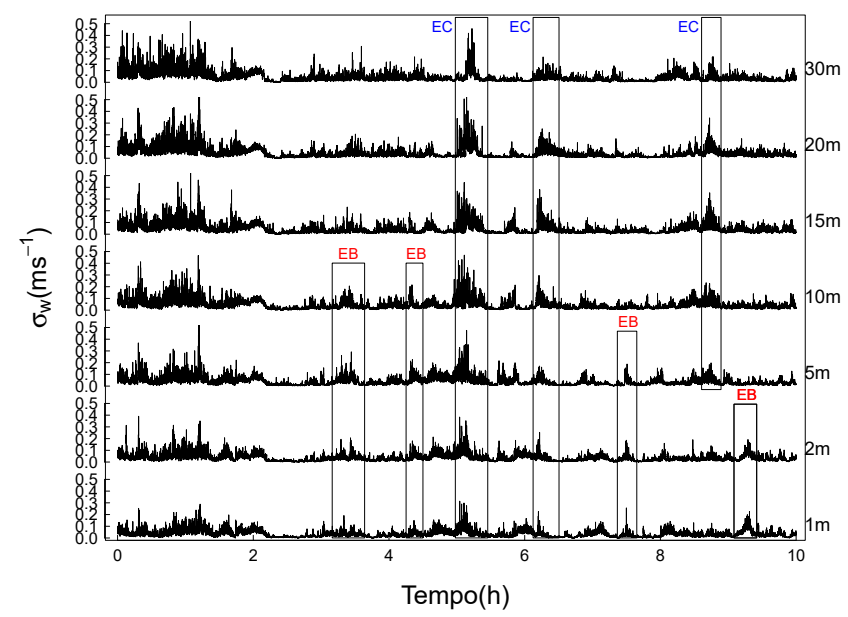

Figura 1: Desvio padrão da velocidade vertical do vento para os 7 níveis da torre para a noite de 06/07 de Fevereiro. Os Eventos de Baixo marcados em vermelho e os Eventos de Cima em azul. A série temporal tem inicio as $20 \mathrm{~h}$ e se estende até as $6 \mathrm{~h}$, no horário local, totalizando um total de 10 horas que estão indicadas na figura.

zado em Coulter e Doran (2002), para a identificação do sentido de propagação dos evento. Além disso, eventos originados em níveis intermediários não foram levados em consideração. A Figura 1, mostra eventos gerados em superfície são menos intensos, mais difíceis de identificar, e muitas vezes não chegam até o topo da torre, enquanto que os EC são mais intensos, ocorrem com maior frequência e na maioria da vezes são suficientes para tornar toda a CLE conectada. Ainda é importante destacar que estrutura vertical da turbulência, mostrada na Figura 1, se repete na maioria das noite (figura não mostrada).

\subsection{Cálculo dos termos de balanço de ECT}

Para todas as noites analisadas, foi calculado o balanço de ECT, com o intuito de identificar quais os termos dominantes durante os diferentes regimes e principalmente, qual o papel de cada termo durante a ocorrência dos eventos. Para o cálculo dos termos da equação de balanço de ECT (equação 1) foi utilizada seguinte metodologia:

Cálculo da tendência de ECT (Termo I): $(\partial \bar{e} / \partial t)$; onde $\bar{e}=\frac{1}{2}\left(\overline{u^{\prime 2}}+\overline{v^{\prime 2}}+\overline{w^{\prime 2}}\right) ; u$ é o componente zonal do vento, $v$ o componente meridional e $w$ é componente vertical do vento. $\partial t$ representa a variação no tempo, usado aqui como 2 minutos (120s). As barras indicam a parte média e as linhas indicam as perturbações das variáveis.
Cálculo produção/destruição de ECT devido ao fluxo na forma de calor (Termo II): $(g / \bar{\Theta})\left(\overline{w^{\prime} \theta_{v}{ }^{\prime}}\right)$; onde $g$ é a gravidade e $\Theta$ é a temperatura potencial virtual de referência, porém, por simplicidade, foi utilizado aqui a temperatura normal em (K). A noite este termo age na destruição de ECT, por isso será chamado, aqui, na maioria das vezes será referido como termo de destruição de ECT.

Cálculo do termo de produção mecânica (Termo III): $\left(-\overline{u^{\prime} w^{\prime}}(\partial \bar{u} / \partial z)-\overline{v^{\prime} w^{\prime}}(\partial \bar{v} / \partial z)\right) ;$ onde $u, v$ e $w$ também são as componentes do vento e $\partial z$ é variação na vertical entre os níveis da torre.

Cálculo do Transporte de ECT (Termo IV): $\left(\partial \overline{w^{\prime} e^{\prime}} / \partial z\right)$; onde $\partial z$ é variação na vertical e $\bar{e}=\left(u^{\prime 2}+v^{\prime 2}+w^{\prime 2}\right)^{1 / 2}$. A obtenção do termo de transporte devido as flutuações de pressão não foi possível devido a inexistência de dados de pressão necessários para realizar o cálculo.

Cálculo da Dissipação (VI): Para o cálculo da dissipação foi feito primeiramente a rotação de coordenadas para que o vetor vento fique alinhado com o eixo $x$. Em seguida, o espectro foi obtido através da Transformada rápida de Fourier (FFT, do inglês fast Fourier transform) e a dissipação foi calculada em intervalos de 120s (2min).

De acordo com Yadav et al. (1996) o espectro em função da frequência é dado por:

$$
S(f)=\alpha\left(\frac{U}{2 \pi}\right)^{2 / 3} \varepsilon^{2 / 3} f^{-5 / 3}
$$

assim, o calculo da dissipação foi realizado conforme descrito em Piper e Lundquist (2004) e Lundquist et al. (2004):

$$
\varepsilon=\frac{2 \pi}{U}\left[\frac{f^{5 / 3} S_{u}(f)}{\alpha}\right]^{3 / 2},
$$

onde $S\left(f u_{i}\right)$ é a frequência do espectro, $U$ é o vetor vento e $\alpha=0,53$ é a constante de Kolmogorov. Ademais, de acordo com Oncley et al. (1996) e Piper e Lundquist (2004), este cálculo pode ser utilizado desde que se tenha dados de alta frequência suficiente para medir velocidades no sub-intervalo inercial. Dessa forma, é feito o ajuste, através do método de mínimos quadrados, para a relação 2 ao espectro experimental (utilizando dados reais) e assim é obtida a dissipação.

Os termos de produção mecânica (PM) e de transporte de ECT, requerem o cálculo de gradientes, desta forma, estes foram obtidos para o cada ponto médio entre os níveis de aferimento na torre. Dessa forma, como a torre possui sete níveis, o primeiro e o último foram perdidos, restando então 5 níveis. Assim, para manter a coerência, o mesmo procedimento foi adotado para os outros termos, ficando dessa forma todos os termos de ECT com a mesma resolução vertical. 


\section{Resultados}

A partir da análise das séries temporais das 108 noites, foram obtidos um total de 61 eventos, 35 EC e 26 EB. Será apresentado aqui três desses eventos intermitentes originados em superfície, dois durante a noite de 06/07 de Fevereiro e um em 27/28 de Fevereiro. Para uma caracterização da estrutura da ECT, durante o surgimento do evento será feito analisando os perfis verticais dos termos da ECT.

A Figura 2-a mostra a série temporal para a ECT, no nível localizado à $2 \mathrm{~m}$ do solo durante o primeiro evento ocorrido em 27/28 de Fevereiro. Para o cálculo dos perfis médios dos termos da equação de balanço de ECT, foi utilizado uma janela temporal que compreende o período que vai desde 6 min antes do evento até o instante no qual o evento alcança a metade de sua ocorrência (indicada pela caixa cinza). Apesar da produção mecânica ser intimamente relacionada com a ECT, os perfis médios dos termos de destruição, transporte e de dissipação mostram que quando somados, nos níveis inferiores, eles são superiores ao termo de PM. Neste caso, além do aumento local da ECT devido a produção mecânica está, provavelmente, ocorrendo também o transporte horizontal de turbulência, por advecção e também o transporte por flutuações de pressão, pois o termo de tendência é positivo. Entretanto, é importante destacar, que estes termos não foram calculados por falta de informação.

A análise dos perfis médios de ECT para o segundo evento (Figura 3), é feita da mesma forma que para o evento apresentado na Figura 2. Este evento possui uma estrutura vertical totalmente distinta do evento anterior, principalmente junto à superfície. Próximo ao solo, o termo dominante é o termo de PM, que é bem maior que os termos de destruição e dissipação como indicado pelo termo de soma. $\mathrm{O}$ termo de transporte é positivo nos níveis inferiores, inciando que existe o transporte de turbulência para baixo. Este resultado está sendo influenciado pelo fato que a intensidade turbulenta é maior no nível de $2 \mathrm{~m}$ que no nível de $1 \mathrm{~m}$ e maior no nível de $5 \mathrm{~m}$ que no nível de $2 \mathrm{~m}$. Nos níveis mais elevados, o aumento da tendencia indica que um outro processo, além da produção mecânica, está atuando na geração de ECT, assim, como ocorreu próximo à superfície no evento anterior.

Para o terceiro evento,(Figura 4), a análise dos termos da equação de balanço de ECT mostra claramente que o termo de PM é bem maior que todos outros termos em todos os níveis mais baixos com exceção do primeiro nível de análise, onde a dissipação de ECT é o maior termo, como indicado pelo termo de soma.
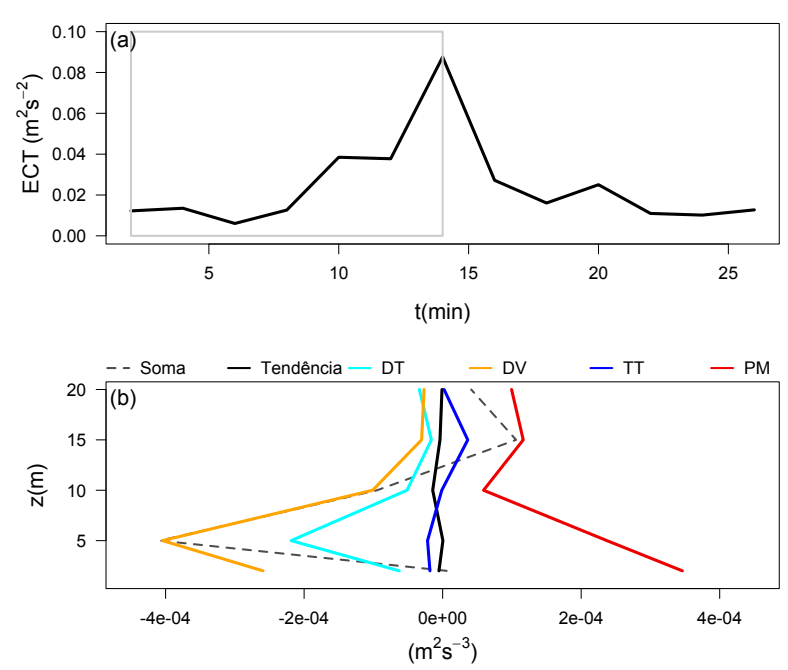

Figura 2: Evolução temporal da ECT em $2 \mathrm{~m}$, para o período na qual ocorre o primeiro evento analisado da noite de 06/07 de Fevereiro. A linha cinza ilustra o período utilizado para o cálculo dos perfis verticais apresentados no painel inferior, que inicia 6 min antes da ocorrência do evento. A Figura 2-b, apresenta os perfis verticais com os termos do balanço de ECT, indicados pela legenda. O termo soma representa a soma dos termos de PM, destruição, transporte turbulento e dissipação viscosa de ECT.

\section{Conclusões}

Alcançar o fechamento exato do balanço de ECT é uma tarefa praticamente impossível a partir de dados reais, devido a grande variabilidade de fenômenos que ocorrem concomitantemente com a turbulência e também influenciam diretamente no escoamento. Dessa forma, a utilização desta abordagem neste trabalho serve para ilustrar e indicar caminhos que possam levar a repostas mais concretas sobre o comportamento da turbulência na CLE muito estável durante eventos intermitentes que são responsáveis pelo transportes de quantidades de baixo para cima (EB).

A partir da identificação dos eventos, verificou-se que os que são originados próximo à superfície, e se propagam para cima, são muito fracos em relação a aqueles gerados nos níveis superiores da CLE, onde a maioria chegou somente até a metade da camada da torre. Através dos perfis dos termos de ECT foi possível ser feito uma melhor caracterização do comportamento da turbulência durante o surgimento dos eventos. Foram apresentadas análises de três casos específicos. Em dois deles não ocorreu a propagação da turbulência até o topo da torre. Para o primeiro os perfis médios dos termos 

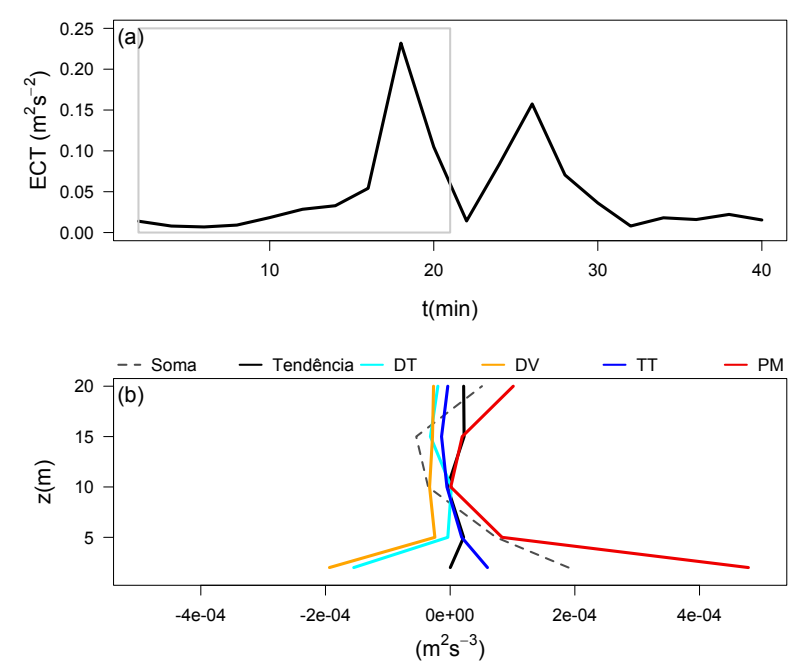

Figura 3: Evolução temporal da ECT em $2 \mathrm{~m}$, para o período na qual ocorre o segundo evento analisado da noite de 06/07 de Fevereiro. A linha cinza ilustra o período utilizado para o cálculo dos perfis verticais apresentados no painel inferior, que inicia 6 min antes da ocorrência do evento. A Figura 3-b, apresenta os perfis verticais com os termos do balanço de ECT, indicados pela legenda. $\mathrm{O}$ termo soma representa a soma dos termos de PM, destruição, transporte turbulento e dissipação viscosa de ECT.

de destruição, transporte e de dissipação mostraram que quando somados, nos níveis inferiores, eles são superiores ao termo de PM. No segundo evento o termo dominante é o termo de PM, que é bem maior que os termos de destruição e dissipação como indicado pelo termo de soma. Para os níveis mais altos o aumento da tendência de ECT indicou que um outro processo, além da PM, está atuando na geração de ECT. Assim, como ocorreu próximo à superfície no primeiro evento. Já o terceiro evento, foi bem mais intenso e se estendeu até os níveis mais altos da torre e a PM também foi maior que os outros termos, com exceção do primeiro nível de análise, onde a dissipação de ECT foi o maior termo.

Dessa forma, as análises feitas através da estrutura vertical da turbulência e do balanço de ECT mostraram que com o aumento do cisalhamento em superfície, a intensidade da turbulência também aumentou nesta região, e esta foi transportada verticalmente para cima pelo termo de transporte turbulento de ECT. O próximo passo deste trabalho é identificar as causas dos eventos que se propagam para cima, e procurar as mesmas características aqui identificadas em dados oriúndos de outros sítios experimentais.
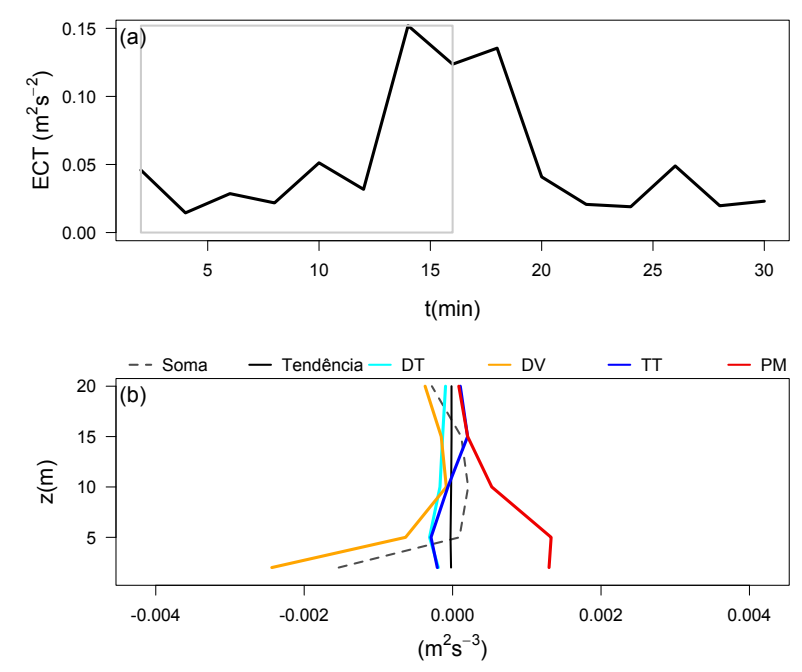

Figura 4: Evolução temporal da ECT em $2 \mathrm{~m}$, para o período na qual ocorre o evento analisado da noite de 27/28 de Fevereiro. A linha cinza ilustra o período utilizado para o cálculo dos perfis verticais apresentados no painel inferior, que inicia $6 \mathrm{~min}$ antes da ocorrência do evento. A Figura 4-b, apresenta os perfis verticais com os termos do balanço de ECT, indicados pela legenda. O termo soma representa a soma dos termos de PM, destruição, transporte turbulento e dissipação viscosa de ECT.

\section{Referências}

Banta, R., Newsom, R., Lundquist, J., Pichugina, Y., Coulter, R., Mahrt, L. (2002). Nocturnal low-level jet characteristics over kansas during cases-99. BoundaryLayer Meteorology, 105(2), 221-252.

Banta, R. M., Pichugina, Y. L., Brewer, W. A. (2006). Turbulent velocity-variance profiles in the stable boundary layer generated by a nocturnal low-level jet. Journal of the atmospheric sciences, 63(11), 2700-2719.

Coulter, R. L., Doran, J. (2002). Spatial and temporal occurrences of intermittent turbulence during cases99. Boundary-layer meteorology, 105(2), 329-349.

Cuxart, J., Yagüe, C., Morales, G., Terradellas, E., Orbe, J., Calvo, J., Fernández, A., Soler, M., Infante, C., Buenestado, P., et al. (2000). Stable atmospheric boundary-layer experiment in spain (sables 98): a report. Boundary-layer meteorology, 96(3), 337-370.

Lundquist, J. K., Piper, J. K., Kosovic, B. (2004). Turbulence kinetic energy budgets and dissipation rates in disturbed stable boundary layers. Boundary-Layer Meteorology, 79, 205-223. 
Mahrt, L. (1999). Stratified atmospheric boundary layers. Boundary-Layer Meteorology, 90, 375-396.

Mahrt, L. (2010). Common microfrentes and other solitary events in the nocturnal boundary layer. BoundaryLayer Meteorology, pp. 1712-1722.

Mahrt, L., Vickers, D. (2006). Extremely weak mixing in stable conditions. Boundary-layer meteorology, 119(1), 19-39.

Oncley, S. P., Friehe, C. A., Businger, J. A., Itsweire, E. C., LaRue, J. C., Chang, S. S. (1996). Surface layer fluxes, profiles and turbulence measurementes over uniform terrain under near-neutral conditions. Atmosphere Science, 53, 1029-1044.

Piper, M., Lundquist, J. K. (2004). Surface layer turbulence measurements during a frontal passage. Journal of the Atmospheric Sciences, 61, 1768-1780.

Stull, R. B. (1988). An Introduction to Boundary Layer Meteorology. Kluwer Academic Publishers, Dordrecht, The Netherlands.

Sun, J., Lenschow, D. H., Burns, S. P., Banta, R. M., Newsom, R. K., Coulter, R., Frasier, S., Ince, T., Nappo, C., Balsley, B. B., Jensen, M., Mahrt, L., Miller, D., Skelly, B. (2004). Atmospheric disturbances that generate intermittent turbulence in nocturnal boundary layers. Boundary-Layer Meteorology, 110(2), 255-279.

Yadav, A. K., Raman, S., Sharan, M. (1996). Surface layer turbulence spectra and dissipation rates during low winds in tropics. Boundary-Layer Meteorology, 79, 205223. 